\title{
ÉDITORIAL
}

\section{Évolution des réglementations en matière de produits phytosanitaires. Positions de l'O.M.S., de l'E.P.A. et de la C.E.E.}

\author{
Michèle RIZET
}

\section{I. - INTRODUCTION}

L'objectif technique d'une directive de qualité concernant les eaux de consommation est de garantir que celles-ci répondent à la fois à des critères de confort et de santé.

Pour cela, la plupart des directives actuellement en vigueur comporte des critères globaux ou organoleptiques traduisant la perception du consommateur et des paramètres très spécifiques visant des molécules ou des groupes de molécules dont les effets néfastes sur la santé ont été démontrés.

Concernant le chapitre des substances titrées dans la réglementation européenne «Paramètres toxiques», les «pesticides» ou produits phytosanitaires occupent une place à part en raison du caractère très artificiel de ce regroupement.

Les caractères communs de ces molécules se résument essentiellement dans leurs effets destructeurs vis à vis d'organismes vivants. Compte tenu de l'extrême variété des organismes cibles et de la grande variété des voies de pénétration ou d'action de ces molécules, elles vont avoir, vis à vis d'une espèce unique Homo Sapiens, des effets d'une nature et d'une intensité très variables.

La présence de produits phytosanitaires dans l'eau peut également en altérer les qualités organoleptiques, toutefois, en raison des normes de principe ou sanitaire imposées, la gène organoleptique due à la présence de pesticides n'est 
constatée que lors de pollutions massives accidentelles. Dans ces cas, l'anomalie de goût, de couleur et/ou de saveur est un excellent signal d'alarme.

Hors des situations accidentelles, la présence de produits phytosanitaires dans les eaux doit répondre à des impératifs réglementaires. Dans les années précédant la nouvelle réglementation de mise sur le marché de produits phytosanitaires, la prolifération des demandes d'autorisation était telle qu'actuellement, on estime à plus de neuf cents le nombre de produits autorisés.

Devant un nombre aussi important de produits et en croissance permanente, on se rend compte de la complexité du travail du législateur et des organismes chargés de la surveillance de la qualité des eaux et du respect de la réglementation.

Par ailleurs - on va le voir - on songe fortement, et la réglementation devrait pouvoir suivre, à imposer des limites intégrant d'éventuels effets de synergie. On n'ose pas évaluer ce que pourrait être la prise en compte des effets interactifs de plusieurs centaines de substances.

Enfin, les résidus de produits phytosanitaires dans l'environnement sont:

- soit réfractaires à la biodégradation, et donc à considérer en tant que tel pendant parfois des délais de plusieurs années,

- soit biodégradables et donnent alors naissance à des produits de dégradation en cascades dont la nature va dépendre des conditions de milieu, de la nature des organismes impliqués.

Ces produits de biodégradation catabolites et métabolites peuvent avoir à leur tour des effets toxiques.

La complexité du sujet ne fait que croître, car il est mis actuellement sur le marché des produits phytosanitaires dits à effet retard. Ce ne sont que les produits de dégradation qui auront l'action phytosanitaire recherchée.

\section{II. - OPTIONS ACTUELLES DES LÉGISLATIONS EXISTANTES}

\section{1. Recommandations de l'OMS, Edition de 1994}

Les recommandations de l'OMS comme l'indique le titre n'ont pas de valeur légale impérative. Elles s'adressent aux responsables de la qualité de l'eau du monde entier. Elles s'appuient sur des études toxicologiques malheureusement souvent peu nombreuses. Elles s'élaborent par le travail d'experts qui se réunissent régulièrement pour consulter les dossiers scientifiques rassemblés émanant du monde entier, discuter les résultats consultés, et proposer des compromis et des calculs différents suivant la nature du problème identifié et l'ensemble des sources de contamination dont l'homme est la cible. 
Dans l'édition de 1994, 35 molécules sont citées dont 19 herbicides, 10 insecticides et 6 produits à usages divers, fongicides par exemple.

La très grande majorité des documents pris en compte concerne des résultats d'études de toxicité sur animaux, souris, rats, lapins, chiens... Les facteurs d'incertitude interviennent pour tenir compte des variations interspécifiques et intraspécifiques généralement $\times 100$ ou x 1000 exceptionnellement $\times 10000$ (cas du 1.2 Dichloropropane) quand les facteurs d'incertitude sont plus nombreux.

Les valeurs servant de base au calcul sont la DSEIO (Dose Sans Effet Indésirable Observé), la DMEIO (Dose Minimale ayant un Effet Indésirable Observé). Pour les produits cancérogènes, le calcul est basé sur le risque $10^{-5}$, c'est-à-dire sur une vie entière l'apparition d'un cas de cancer sur 100000 personnes.

Le calcul est enfin affecté d'un pourcentage représentant la part des apports attribués à l'eau. Ce facteur varie de 1 à $10 \%$. Le choix de l'un ou de l'autre ne paraît pas toujours parfaitement étayé.

Le principe de précaution n'est pas véritablement appliqué dans le sens que seules les molécules ayant une toxicité démontrée au sens large (cytotoxicité et/ou mutagénéicité, et/ou cancérogénicité et/ou action mesurable sur des organes, foie, rein...) sont prises en compte. Quand le dossier apparaît insuffisant aux experts, aucune valeur limite n'est recommandée. La molécule concernée est affectée de la note: «données insuffisantes ».

Compte tenu de la lourdeur des structures mises en place, la remise à jour des recommandations de l'OMS demande environ dix ans.

\section{2. Réglementation de l'Agence Américaine pour la Protection de l'Environnement (USEPA)}

La réglementation concerne un ensemble de contaminants organiques essentiellement pesticides et solvants au nombre de 50 environ. L'USEPA base ses MCL (Niveau Maximum de Concentration) sur des travaux disponibles dans le monde et des études toxicologiques financées par ses soins.

Les valeurs énoncées par l'USEPA ont un caractère de respect obligatoire. Elles sont souvent à des niveaux de concentration différents des recommandations OMS.

Exemple:

$\begin{array}{lll} & \text { U.S. } & \text { O.M.S. } \\ \text { Alachlore } & 2 \mu \mathrm{g} / 1 & 2 \mu \mathrm{g} / 1 \\ \text { Heptachlore } & 0.4 \mu \mathrm{g} / 1 & 0.03 \mu \mathrm{g} / 1 \\ \text { Lindane } & 0.2 \mu \mathrm{g} / 1 & 2 \mu \mathrm{g} / 1 \\ \text { Simazine } & 4 \mu \mathrm{g} / 1 & 2 \mu \mathrm{g} / 1\end{array}$

Les normes américaines sont en constante évolution. Des études toxicologiques peuvent permettre, à tout moment, de reconsidérer une valeur. Actuelle- 
ment, un complément d'investigation est en cours sur l'atrazine pour reconsidérer l'éventualité d'un pouvoir cancérogène.

\section{3. Directives européennes et leur intégration dans la législation française}

La directive européenne actuellement en vigueur 80/778 du 15 juillet 1980 présente un paramètre $\mathrm{n}^{\circ} 55$ dit «Pesticides et Produits apparentés». Contrairement à d'autres paramètres, aucune valeur ne figurait dans la colonne des Niveaux Guides, la règle étant que, pour tous les toxiques, le Niveau Guide était une absence.

Les Concentrations Maximales Admissibles (CMA) sont des limites impératives imposées à toute la Communauté Européenne.

Aucune liste de pesticide n'est indiquée. Seule figure une définition:

On entend par pesticides et produits apparentés:

- les insecticides organo-chlorés persistant, organophosphorés, carbamates,

- les herbicides,

- les fongicides,

- les PCB et PCT.

La CMA imposée est simple: $0.1 \mu \mathrm{g} / \mathbf{l}$ par substance individualisée et $0.5 \mu \mathrm{g} / \mathrm{l}$ au total.

On peut juger de la singularité de cette réglementation par rapport à celles précédemment décrites.

La France n'a pu qu'adopter cette réglementation en précisant toutefois des valeurs plus contraignantes:

- aldrine et dieldrine $=0.03 \mu \mathrm{g} / 1$

- Heptachlore et Heptachlore epoxyde $=0.03 \mu \mathrm{g} / 1$

Ces valeurs sont conformes aux recommandations de l'OMS.

Par contre, la règle européenne est l'interdiction aux Etats d'adopter des valeurs moins contraignantes que celles imposées à l'échelle européenne.

La directive européenne 80/778 a donc actuellement dix-sept ans. Beaucoup de connaissances ont évolué, un grand besoin de révision s'est fait sentir. Une directive nouvelle est sur le point de voir le jour. 


\section{III. - TENDANCES DE L'ÉVOLUTION DE CES RÉGLEMENTATIONS}

Des équipes dans le monde, notamment aux Etats-Unis, au Japon et en Europe, travaillent en permanence sur les molécules générées par l'activité humaine et aboutissant dans les eaux. Les disciplines concernées sont l'analyse chimique, la toxicologie, et, à moindre titre malheureusement, l'épidémiologie.

En ce qui concerne les produits phytosanitaires, le nombre de molécules homologuées est sans cesse grandissant. Il est tel, actuellement de l'ordre de neuf cents molécules, qu'il n'est pas possible de laisser leur contrôle et leur recherche au hasard.

C'est pour orchestrer cet ensemble Fabrication/Usage/Contrôle que le Ministère de l'Environnement à demandé aux préfets de région d'organiser la concertation au sein des régions, et que la Communauté Européenne établit des règles plus strictes de mise sur le marché des produits phytosanitaires avec la publication au Journal Officiel des C.E. au 27 septembre 1997 de l'annexe VI (97/57/CE) de la Directive 91/414 /CEE.

Cette directive du Conseil du 22 septembre 1997, donne les principes pour l'évaluation et l'autorisation des produits phytopharmaceutiques. Elle traite aussi bien de l'impact du produit et de ses dérivés sur la faune et la flore, que de ses propriétés physico-chimiques et des moyens analytiques à mettre en cuvre pour en assurer le contrôle dans l'environnement.

A la page $265 / 96$ de cette directive, sont envisagés les contacts possibles avec les eaux de surface et les eaux souterraines qui doivent être étudiés en détail dans les zones d'utilisation potentielle des produits. Une des carences du système paraît être le prix de revient élevé des analyses préconisées. Ceci est un frein à la multiplication judicieuse des contrôles dans le temps et dans l'espace.

A l'énoncé de ces dispositions, on saisit toute la prise de conscience des difficultés engendrées par l'emploi actuel de produits multiples, à l'évolution non maîtrisées, à l'analyse réservée à quelques laboratoires très spécialisés, puissamment équipées des industries phytopharmaceutiques.

Devant cette situation, les règlements concernant les eaux de consommation doivent pouvoir être révisables à tout moment. L'Organisation Mondiale de la Santé travaille constamment à collecter les données toxicologiques et épidémiologiques publiées dans le monde pour préparer une nouvelle édition des recommandations.

L'Agence Américaine pour l'Environnement mène des recherches sur les dossiers insuffisamment documentés. Actuellement, l'Atrazine fait l'objet d'un complément d'étude pour vérifier l'existence ou non d'un caractère cancérogène de la molécule. 
La directive européenne 80/778/CEE est en profonde révision depuis plusieurs années. La lourdeur de la machine européenne rend actuellement très difficile une bonne rapidité de remise à jour par un va et vient de longue durée entre les instances techniques et politiques. Le projet de directive disponible déjà depuis plusieurs années (28-04-95) a fait l'objet de nombreux amendements de la part des députés du Parlement Européen.

Concernant les pesticides, ils ne font pas l'objet d'une évolution notoire. La valeur individuelle de $0.1 \mu \mathrm{g} / 1$ est maintenue quelle que soit la molécule considérée.

La valeur globale de $0.5 \mu \mathrm{g} / \mathrm{l}$ avait été écartée du projet mais sous la poussée de plusieurs délégations, elle sera rétablie (selon le document COM (97) $228 \mathrm{du}$ 4 juin 1997, en note 5 bis de la partie B de l'annexe I).

De même, la note 5 point $a$ ) modifiée devient :

Par pesticides, on entend:

les insecticides organiques,

les herbicides organiques,

les fongicides organiques,

les nématocides organiques,

les acaricides organiques,

les algicides organiques,

et autres produits apparentés (régulateurs de croissance et métabolites qui sont des pesticides).

\section{Le point b) devient:}

Seuls les pesticides dont la présence dans une distribution donnée est probable doivent être contrôlés.

Le point d) attestant l'examen par la commission de valeurs individuelles à fixer pour une substance donnée, après évaluation des informations scientifiques disponibles, disparaît.

Après une timide avancée vers une norme technique modulée en fonction de la nature des produits et de leur impact vis à vis de la santé scientifiquement démontré, on en revient à une position de principe du $0.1 \mu \mathrm{g} / 1$ quelle que soit la molécule considérée.

Par contre, la recommandation de ne contrôler que les pesticides dont la présence est probable, est intéressante. Elle implique un rapprochement impératif entre ceux dont les activités gravitent autour des produits phytopharmaceutiques: fabricants, commerçants, utilisateurs, et analystes de l'eau de consommation, et des ressources en eau.

Il est question de mettre en place ultérieurement un système permettant une mise à jour plus rapide des paramètres le nécessitant, sans une remise en question de l'ensemble de la directive. 


\section{IV. - CONCLUSIONS}

On peut conclure à travers le parallèle établi ici entre différentes réglementations :

- La réglementation doit être évolutive pour suivre au plus près la mise sur le marché et l'usage des produits phytopharmaceutiques.

- Le contrôle plus stricte mis en place au niveau européen lors de l'autorisation de mise sur le marché était indispensable et devrait déboucher sur une moindre prolifération de produits nouveaux, des produits mieux ciblés non seulement en fonction de l'usage agricole, mais de l'impact environnemental et de la possibilité du suivi analytique de lui-même et de ses métabolites.

- La directive européenne paraît simpliste avec sa limite unitaire.

- Par contre, les réglementations à structure technique souffrent d'une insuffisance de résultats toxicologiques et épidémiologiques. Ces textes constituent un encouragement très fort à la recherche scientifique dans le domaine environnement et santé.

- L'insuffisance actuelle des connaissances apparaît dans la non concordance des valeurs limites proposées dans les différentes réglementations.

- Un point très important à souligner est la prise de conscience de la nécessité du rapprochement de tous les acteurs concernés pour la mise en place d'un compromis satisfaisant les impératifs des divers usages du milieu. 